\title{
Liposomal lidocaine to improve procedural success rates and reduce procedural pain among children: a randomized controlled trial
}

\author{
Anna Taddio, Herpreet Kaur Soin, Suzanne Schuh, Gideon Koren, Dennis Scolnik
}

ß See related article page 1699

Abstract

Background: Historically, children have been undertreated for their pain, and they continue to undergo painful cutaneous procedures without analgesics. A new topical anesthetic, liposomal lidocaine $4 \%$ cream (Maxilene, RGR Pharma, Windsor, Ont.), has become available. It has pharmacologic properties that are superior to other topical anesthetics, including an onset of action of only 30 minutes. We sought to determine the success rate of cannulation, analgesic effectiveness, procedure duration and rate of adverse skin reactions when liposomal lidocaine is used before intravenous cannulation of children.

Methods: In this double-blind randomized controlled trial, children aged 1 month to 17 years received liposomal lidocaine or placebo before cannulation. Success on first cannulation attempt was recorded, and, among children 5 years and older, pain was evaluated before and after the attempt by the child, parents and research assistant using a validated measure (Faces Pain Scale-Revised). For children younger than 5 years, pain was evaluated by the parents and research assistant only. The total duration of the procedure and adverse skin reactions were also recorded.

Results: Baseline characteristics did not differ $(p>0.05)$ between children who received liposomal lidocaine $(n=69)$ and those who received placebo $(n=73)$. Cannulation on the first attempt was achieved in $74 \%$ of children who received liposomal lidocaine compared with 55\% of those who received placebo $(p=0.03)$. Among children 5 years of age and older $(n=67)$, lower mean pain scores during cannulation were reported by those receiving liposomal lidocaine $(p=0.01)$. Similarly, lower mean pain scores during cannulation were reported by the parents and research assistant for all children who received liposomal lidocaine than for all those who received placebo $(p<0.001)$. The mean total procedure duration was shorter with liposomal lidocaine (6.7 v. 8.5 minutes; $p=0.04)$. The incidence of transient dermal changes was $23 \%$ in both groups $(p=1.0)$.

Conclusions: Use of liposomal lidocaine was associated with a higher intravenous cannulation success rate, less pain, shorter total procedure time and minor dermal changes among children undergoing cannulation. Its routine use for painful cutaneous procedures should be considered whenever feasible.

CMAJ 2005;172(13):1691-5
$\mathrm{P}$ ainful medical procedures are routinely performed on children for diagnostic and therapeutic reasons. The provision of analgesia for these procedures, however, remains uncommon. ${ }^{1}$ Untreated pain has both short-term and long-term consequences. In the short term, there is pain during the actual procedure. This contributes to a lack of cooperation by the child, unsuccessful procedure attempts, repeated attempts, additional pain and a prolonged total procedure time. In the long term, repeated painful procedures can lead to conditioned anxiety responses and increased pain perception., ${ }^{2,3}$ Inadequate analgesia during an initial procedure may diminish analgesic effectiveness at subsequent procedures. ${ }^{4}$ Moreover, there is a relation between painful procedures in childhood and blood-injection-injury phobia,${ }^{5}$ a condition that affects up to $10 \%$ of adults and may cause people to avoid medical care. ${ }^{6}$ In light of the cumulative evidence of the negative consequences of untreated pain in childhood, interventions are needed to diminish pain among children undergoing medical procedures and to facilitate successful completion of procedures.

Intravenous cannulation is a common, painful medical procedure. Although local anesthetics reduce the pain of cannulation, ${ }^{7-11}$ most preparations are not feasible for routine use. The "gold standard" for skin anesthesia, lidocaine-prilocaine $5 \%$ cream, requires a 60 -minute application time. In addition, it causes vasoconstriction, ${ }^{12}$ which potentially obscures landmarks and makes cannulation more difficult. ${ }^{13}$ Another commercially available preparation, amethocaine $4 \%$ gel, requires a 30 -minute application time. However, it frequently causes vasodilatation and may induce hypersensitivity with repeated use..$^{14}$ An alternative option, subcutaneous injection of lidocaine, requires only a few minutes to administer, but it is associated with an extra and painful puncture and is therefore not routinely used. ${ }^{15}$

Liposomal lidocaine 4\% cream $^{16}$ (Maxilene, RGR Pharma, Windsor, Ont.) was launched in Canada in 2003. The liposome-encapsulated formulation protects the anesthetic from being metabolized too quickly. ${ }^{17}$ Liposomal lidocaine has the advantages of "needle-free" administration, a short onset of action and minimal vasoactive properties that min- 
imize any potential interference with cannulation success. It is not associated with methemoglobinemia, a systemic side effect of lidocaine-prilocaine. ${ }^{18}$

Among children, liposomal lidocaine is as effective as lidocaine-prilocaine for decreasing pain from venipuncture ${ }^{19}$ and intravenous cannulation, ${ }^{20,21}$ and as effective as buffered lidocaine injection for decreasing intravenous cannulation pain. ${ }^{15}$ Previous studies have not compared liposomal lidocaine with placebo. We conducted such a comparison to determine whether liposomal lidocaine improves cannulation success rates. We also sought to determine whether it reduces pain and procedure duration and is associated with a low frequency of dermal reactions.

\section{Methods}

This double-blind randomized controlled trial was conducted in the emergency department of The Hospital for Sick Children in Toronto. The hospital research ethics board approved the protocol, and informed written consent was obtained from all parents and written assent from children over 7 years of age.

Children 1 month to 17 years who required a peripheral intravenous cannula were eligible. Children with critical illness requiring immediate cannulation (e.g., sepsis, severe dehydration) or known or suspected hypersensitivity to local anesthetics, who were already receiving opioid analgesia or topical anesthesia or who had previously participated were excluded.

Ferndale Laboratories (Ferndale, Mich.) provided 4\% liposomal lidocaine and placebo (base without active ingredient) in $5-\mathrm{g}$ tubes. The creams and tubes were visually identical. The tubes were individually dispensed by the research pharmacist after being labelled with a sequential code number according to a randomization table that assigned each child to liposomal lidocaine or placebo in blocks of 4 . The table was stored in a secure location.

After consent was obtained, the nurse chose 2 sites where she would attempt cannulation. Each subject received $1 \mathrm{~g}$ of either ac- tive or placebo drug at both sites for 30 minutes under occlusion with Tegaderm or IV 3000. After 25 minutes, using the Faces Pain Scale-Revised (FPS-R), ${ }^{22}$ the research assistant rated the child's baseline pain and obtained baseline pain scores from all parents and from children who were 5 years of age or older. The FPS-R provides a way to measure pain among children using pictures of 6 facial expressions that correspond to increasing pain intensity from "no pain" to "worst possible pain" and that are scored from 0 to 5 . Child and parent understanding of the FPS-R was pretested. The face that best described the amount of pain currently experienced was selected, and the corresponding numerical value was used in the analysis. A standardized dialogue was used, as obtained from the Pediatric Pain Web site of the Pediatric Pain Research Lab at Dalhousie University (www.dal.ca/ pedpain/).

The cream was removed 30 minutes after application, and skin reactions were recorded. The nurse rated the level of difficulty expected for cannulation on a 5-point scale that ranged from extremely easy to easy, average, difficult and extremely difficult. The nurse then inserted the cannula.

The primary outcome was successful intravenous insertion on the first attempt. Successful cannulation was recorded when the skin was breached only once and the intravenous line remained in place for at least 5 minutes (to account for extravasation). A failure was recorded if a second puncture was required or if a site other than 1 of the 2 chosen sites was cannulated. The number of attempts and the duration of the procedure (from first puncture to the secure of a working intravenous cannula) were recorded.

After the first attempt, whether or not it was successful, the research assistant rated child pain and obtained pain scores from children 5 years and older and from all parents using the FPS-R. Pain during cannulation was calculated as the pain score after the first cannulation attempt minus the baseline score.

On the basis of an estimated $65 \%$ success rate for cannulation in our emergency department, we determined that 144 children were needed to detect a $20 \%$ difference in the success rate for the local anesthetic with $\alpha=0.05$ and $\beta=0.2$. The analysis plan was a modified intent-to-treat approach that excluded chil-

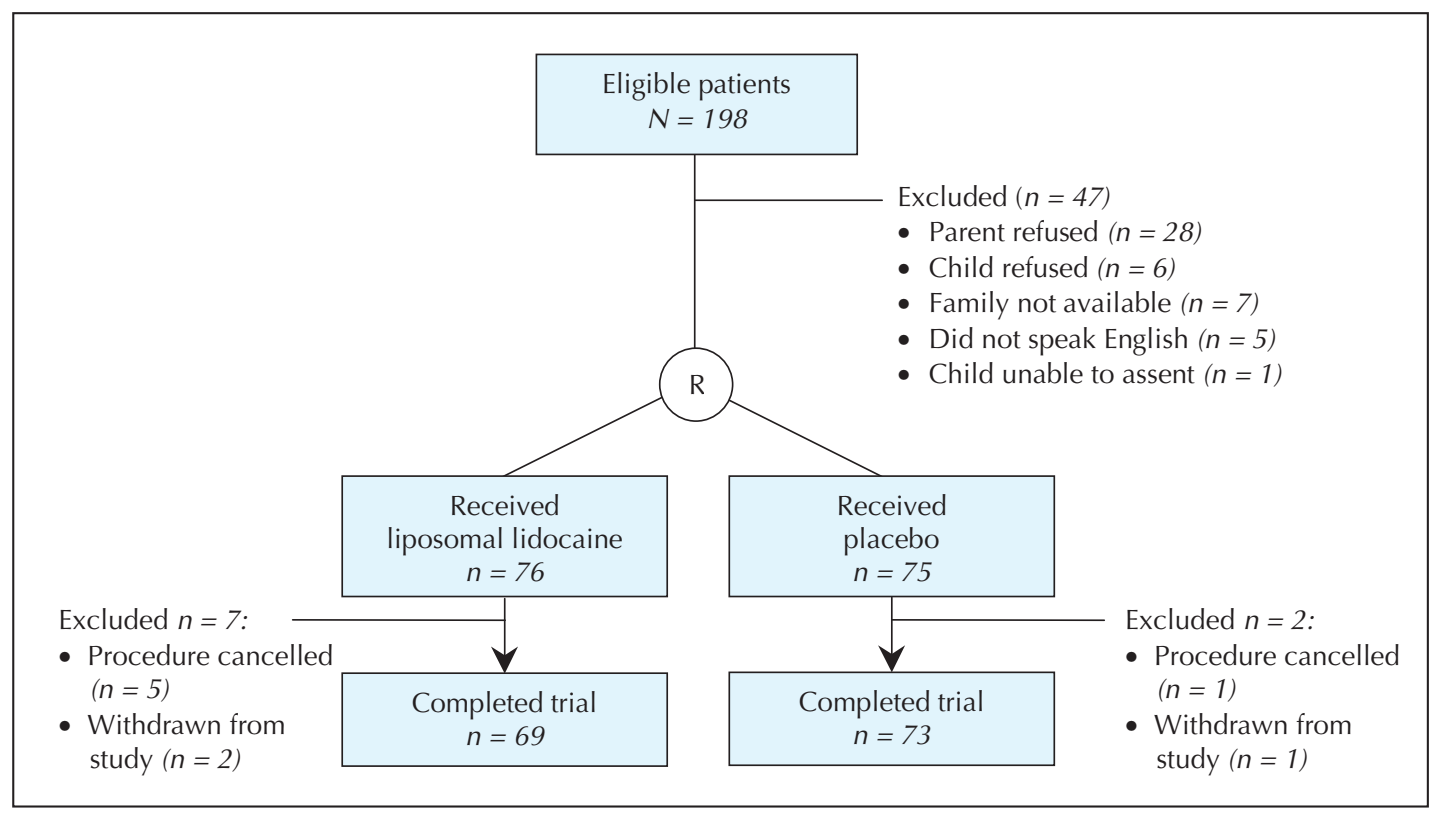

Fig. 1: Flow of children through the trial. 
dren for whom the study drug was discontinued for reasons unrelated to efficacy and children who withdrew from the study. Differences in categorical data and continuous data were compared using $\chi^{2}$ test and $t$ test. Logistic and linear regression analyses, respectively, were used to determine the effects of group assignment, sex and age of the patient, years of nursing experience, and nurses' assessment of cannulation difficulty on cannulation success and pain. A p value of 0.05 or less was considered significant.

\section{Results}

Recruitment occurred from June 2003 to February 2004. A total of 142 children completed the trial, of whom 69 received liposomal lidocaine and 73 received placebo (Fig. 1). There were no significant differences in baseline characteristics between the 2 study groups (Table 1).

All first cannulation attempts were made at a site that was pretreated with either liposomal lidocaine or placebo. The cannulation success rate was significantly higher among children who received liposomal lidocaine than among those who received placebo $(74 \%$ v. $55 \% ; p=$ 0.03 ) (Table 2). The total procedure time required to achieve successful cannulation was shorter among children who received liposomal lidocaine (6.7 minutes v. 8.5 minutes; $p=0.04$ ) (Table 2). The incidence of transient skin reactions was the same in both groups $(23 \% ; p=1.0)$ (Table 2).

Table 1: Baseline characteristics of children participating in the trial

\begin{tabular}{|c|c|c|c|}
\hline \multirow[b]{2}{*}{ Characteristic } & \multicolumn{2}{|c|}{ Group; value } & \multirow[b]{2}{*}{$p$ value } \\
\hline & $\begin{array}{l}\text { Liposomal } \\
\text { lidocaine } \\
(n=69)^{*}\end{array}$ & $\begin{array}{l}\text { Placebo } \\
(n=73)\end{array}$ & \\
\hline \multicolumn{4}{|l|}{ Demographic } \\
\hline Age, mean (SD), yr & $6.7(5.1)$ & $5.1(4.8)$ & 0.07 \\
\hline Male, no. $(\%)$ & $34(49.3)$ & $45(61.6)$ & 0.19 \\
\hline Weight, mean (SD), kg & $29.0(21.7) \dagger$ & $24.1(18.0)$ & 0.14 \\
\hline Clinical & & & 0.65 \\
\hline Fever or infection, no. & 11 & 18 & \\
\hline Gastrointestinal, no. & 18 & 13 & \\
\hline Respiratory, no. & 12 & 10 & \\
\hline Pain or trauma, no. & 11 & 12 & \\
\hline Other, no. $\neq$ & 17 & 20 & \\
\hline Reason for IV cannulation & & & 0.58 \\
\hline Medication administration, no. & 33 & 33 & \\
\hline Hydration, no. & 18 & 16 & \\
\hline $\begin{array}{l}\text { Patient potentially required IV } \\
\text { fluid or medication, no. }\end{array}$ & 18 & 24 & \\
\hline Site, dorsum of hand, no. (\%) & $64(93)$ & $63(86)$ & 0.33 \\
\hline
\end{tabular}

Notes: $\mathrm{SD}=$ standard deviation, $\mathrm{IV}=$ intravenous

*Unless indicated otherwise.

†Data were missing for 1 patient who received liposomal lidocaine.

łIncludes all others (e.g., metabolic conditions, seizures, poison ingestion).
Logistic regression analysis revealed that group assignment and nurses' ratings of expected cannulation difficulty predicted cannulation success. Pretreatment with liposomal lidocaine and a lower difficulty rating were associated with an improved success rate ( $p=0.01$ for both variables). Use of liposomal lidocaine was associated with an odds ratio of 0.38 (95\% confidence interval [CI] 0.18-0.8) of cannulation failure compared with placebo. Conversely, an increase of 1 point on the scale used to rate expected cannulation difficulty was associated with an odds ratio of 1.64 (95\% CI 1.11-2.43) of cannulation failure.

The pain scores are displayed in Table 3. In the subsample of children able to rate their own pain (age $\geq 5$ years, $n=67$ ), those who received liposomal lidocaine reported less pain than those who received placebo $(p=$ 0.01). The parents and research assistant also reported less pain among the children who received liposomal lidocaine $(p<0.001)$. Regression analyses revealed that, for the children's own pain ratings, factors associated with less pain were pretreatment with liposomal lidocaine $(p=0.01)$ and male sex $(p=0.02)$, which explained $16 \%$ of the variance. Parent and research assistant scores were predicted by pretreatment with liposomal lidocaine $(p<0.001)$, which explained $10 \%$ and $19 \%$ of the variance respectively.

\section{Table 2: Measurements of intravenous cannulation} procedures

\begin{tabular}{|c|c|c|c|}
\hline \multirow[b]{2}{*}{ Variable } & \multicolumn{2}{|c|}{ Group; no. (\%)* } & \multirow[b]{2}{*}{$p$ value } \\
\hline & $\begin{array}{l}\text { Liposomal } \\
\text { lidocaine } \\
(n=69)^{*}\end{array}$ & $\begin{array}{l}\text { Placebo } \\
(n=73)^{*}\end{array}$ & \\
\hline $\begin{array}{l}\text { Nurse rating of cannulation } \\
\text { difficulty, mean (SD) } \dagger\end{array}$ & $2.7(0.9)$ & $2.8(1.0)$ & 0.66 \\
\hline \multicolumn{4}{|l|}{ No. of attempts } \\
\hline 1 & $51(74)$ & $40(55)$ & 0.03 \\
\hline 2 & $3(4)$ & $2(3)$ & \\
\hline 3 & $13(19)$ & $23(31)$ & \\
\hline 4 & $2(3)$ & $7(10)$ & \\
\hline 5 & $0(0)$ & $1(1)$ & \\
\hline \multicolumn{4}{|l|}{$\begin{array}{l}\text { Duration of procedure, } \\
\text { mean (SD), min }\end{array}$} \\
\hline First attempt & $\begin{array}{l}(n=69) \\
5.5(2.8)\end{array}$ & $\begin{array}{l}(n=72) \text { 促 } \\
5.4(2.9)\end{array}$ & 0.82 \\
\hline Total procedure & $\begin{array}{l}(n=62) \S \\
6.7(3.6)\end{array}$ & $\begin{array}{l}(n=63) \S \\
8.5(5.9)\end{array}$ & 0.04 \\
\hline \multicolumn{4}{|l|}{ Adverse skin reactions } \\
\hline None & $53(76.8)$ & $56(76.7)$ & 1.0 \\
\hline Blanching & $14(20.3)$ & $13(17.8)$ & \\
\hline Erythma & $2(2.9)$ & $2(2.7)$ & \\
\hline Itchiness & 0 & $2(2.7)$ & \\
\hline
\end{tabular}

Notes: SD = standard deviation

*Unless indicated otherwise.

†The cannulation difficulty score ranged from 1 ("extremely easy") to 5 ("extremely difficult").

fData were missing for 1 patient who received placebo.

$\S$ The procedure was aborted or temporarily suspended by the nurse after a failure at first attempt or after multiple attempts ( $n=7$ for liposomal lidocaine, $n=10$ for placebo). 


\section{Interpretation}

Relative to placebo, use of liposomal lidocaine was associated with $35 \%$ higher cannulation success rates and 21\% shorter overall procedure times. Relative to children who received placebo, children who received liposomal lidocaine had $43 \%$ less pain according to the pain scores of the subset of children who self-reported pain and $35 \%$ and $43 \%$ less pain according to the parent and observer pain scores respectively for all children. The frequency of adverse skin reactions was the same in the 2 groups. We hypothesize that the higher cannulation success rate observed among children who received liposomal lidocaine was due to a reduction in pain and lack of dermal changes. It is likely that because pain during needle puncture was less, the children moved less during cannula placement, which thereby facilitated the procedure. Moreover, the lack of significant dermal changes minimized alterations in skin landmarks. The absence of any reported differences in technical difficulty suggests that the higher success rate in the group that received liposomal lidocaine can be attributed to analgesic use and not other factors.

Our results refute previous claims that topical local anesthesia interferes with procedure success rate. ${ }^{13}$ They also have important implications for the management of procedural pain in children. With this new preparation, it is possible to provide prompt and practical analgesia, improve the likelihood of successful cannulation upon first attempt, and reduce overall procedure time. This, in turn, will result in reduced anxiety for children, parents and health care workers during the cannulation procedure and future procedures. The time saved with the faster comple-

Table 3: Faces Pain Scale-Revised scores during first cannulation attempt*

\begin{tabular}{lccc}
\hline & \multicolumn{2}{c}{ Group; mean (SD) } & \\
\cline { 2 - 3 } & $\begin{array}{l}\text { Liposomal } \\
\text { lidocaine }\end{array}$ & Placebo & $p$ value \\
\hline Child $(\geq \mathbf{5}$ yr only) & $(n=37)$ & $(n=30)$ & \\
Baseline & $1.3(1.2)$ & $1.6(1.6)$ & 0.29 \\
After first attempt & $2.6(1.5)$ & $3.9(1.5)$ & 0.001 \\
Difference & $1.3(1.3)$ & $2.3(1.6)$ & 0.01 \\
Parents & $(n=65) \dagger$ & $(n=67) \dagger$ & \\
Baseline & $1.2(1.2)$ & $1.3(1.2)$ & 0.695 \\
After first attempt & $2.7(1.5)$ & $3.6(1.3)$ & $<0.001$ \\
Difference & $1.5(1.3)$ & $2.3(1.2)$ & $<0.001$ \\
Research assistant & $(n=69)$ & $(n=73)$ & \\
Baseline & $1.1(1.1)$ & $1.1(1.2)$ & 0.912 \\
After first attempt & $2.4(1.4)$ & $3.4(1.3)$ & $<0.001$ \\
Difference & $1.3(1.0)$ & $2.3(1.2)$ & $<0.001$ \\
\hline
\end{tabular}

Note: $\mathrm{SD}=$ standard deviation

*Faces Pain Scale-Revised scores range from 0 ("no pain") to 5 ("worst possible pain"). Pain during cannulation was calculated as the pain score after the first attempt minus the baseline pain score.

†Pain scores were missing for 4 parents whose children received liposomal lidocaine and 6 parents of children who received placebo. tion of the procedure may also allow health care workers more time to attend to other patient issues. Although it is clear from the results of this study that liposomal lidocaine improves procedure success rates, it is not known whether other anesthetic preparations have similar effects. This question must be addressed in future studies that compare different agents.

Of the 151 children participating in the study, $9(6 \%)$ were excluded from the primary analysis. The reasons for exclusion were post-randomization discovery of patient ineligibility $(n=6)$ and withdrawal from the study $(n=3)$. Exclusions made after randomization threaten to bias treatment comparisons. Although details regarding outcomes should be followed up and data analyzed according to group assignment, we nevertheless excluded the 3 children who were withdrawn. It has been suggested that fewer than $5 \%$ loss to follow-up is an acceptable figure that leads to little bias in results. ${ }^{23}$ Another limitation of our study was missing data, including pain scores from parents whose child received liposomal lidocaine $(n=4)$ and parents whose child received placebo $(n=6)$, duration of first cannulation attempt for 1 child who received placebo, and duration of total procedure when the procedure was aborted or temporarily suspended by the nurse after a failure at first attempt or after multiple attempts ( $n=7$ for liposomal lidocaine, $n=10$ for placebo).

The rate of skin colour changes observed in this study was higher than in some previously reported studies involving liposomal lidocaine ${ }^{15,19,20}$ and may reflect differences in assessment techniques. The colour changes, however, were minor in nature and occurred with equal frequency among the children who received lidocaine and those who received placebo, which suggests they were due to hydration of the skin from occlusion and were not clinically significant.

Pain perception is influenced by anxiety, underlying conditions, culture and many other factors. We did not rate child anxiety specifically before cannulation. Rather, we assessed pain, which allowed us to correct for baseline pain in our analysis, and we expected that anxiety would be reflected in baseline pain ratings. We observed no differences in baseline pain between groups, which provides indirect evidence that there was no difference in anxiety levels. The FPS-R was chosen to measure pain intensity because its validity has been established in our patient population..$^{22}$ Faces scales are the preferred method of pain assessment among children, parents and nurses, and they have been validated in the emergency department setting. ${ }^{24-27}$ It should be acknowledged that pain ratings by the parents and research assistant may have been influenced by the child's behavioural distress. In this study, we did not use cointerventions such as behavioural interventions ${ }^{28,29}$ or sedation, ${ }^{30}$ which may augment the analgesic effects of liposomal lidocaine.

In conclusion, liposomal lidocaine improves procedure success rate, reduces pain, reduces procedure time, and is associated with minor dermal changes when used for cutaneous analgesia in children. When combined with the 
drug's relative ease of administration and fast onset of action, these effects make liposomal lidocaine ideally suited for routine use. We recommend that liposomal lidocaine be adopted as the new standard of care for cutaneous analgesia in children.

\section{This article has been peer reviewed.}

From the Departments of Pharmacy (Taddio, Soin) and Pediatrics (Schuh, Koren, Scolnik) and the Research Institute (Taddio, Schuh, Koren, Scolnik), The Hospital for Sick Children, and the Faculties of Pharmacy (Taddio, Soin, Koren) and Medicine (Schuh, Koren), University of Toronto, Toronto, Ont.

Competing interests: None declared.

Contributors: Anna Taddio drafted the article and, with Herpreet Soin and Dennis Scolnik, was responsible for data acquisition. All of the authors contributed to the study concept and design, analysis and interpretation of the data and revision of the article, and approved the final version submitted for publication.

Acknowledgements: We thank Svetlana Khaikin, research nurse in the emergency department, and Jonathan Isenberg, student volunteer, for providing help with aspects of data collection during the conduct of this study.

Anna Taddio is supported by the Canadian Institutes of Health Research (CIHR) New Investigator Award. Gideon Koren is a Senior Scientist of CIHR and holder of the Ivey Chair in Molecular Toxicology, The University of Western Ontario. Ferndale Laboratories provided liposomal lidocaine $4 \%$ cream and placebo study medications. The study was funded in part by the Canadian distributor of liposomal lidocaine 4\% cream, RGR Pharma.

\section{References}

1. Walco GA, Burns JP, Cassidy RC. The ethics of pain control in infants and children. In: Schechter NL, Berde CB, Yaster M, eds. Pain in infants, children, and adolescents. 2nd ed. New York: Lippincott Williams \& Wilkins; 2003. p. 157-68.

2. Taddio A, Shah V, Gilbert-MacLeod C, Katz J. Conditioning and hyperalgesia in newborns exposed to repeated heel lances. FAMA 2002;288(7):857-61.

3. Taddio A. Effects of early pain experience (the human literature). In: McGrath PJ, Finley A, eds. Chronic and recurrent pain in children and adolescents: progress in pain research and management. Vol. 13. Seattle: IASP Press; 1999. p. 57-74.

4. Weisman SJ, Bernstein B, Schechter NL. Consequences of inadequate analgesia during painful procedures in children. Arch Pediatr Adolesc Med 1998;152 (2):147-9.

5. American Psychiatric Association. Specific phobia. In: Diagnostic and statistical manual of mental disorders. 4th ed. Washington: American Psychiatric Association; 2000: p. 443-50.

6. Ost L-G. Blood and injection phobia: background and cognitive, physiological, and behavioral variables. 7 Abnormal Psychol 1992;101:68-74.

7. Hallen B, Uppfeldt A. Does lidocaine-prilocaine cream permit painfree insertion of IV catheters in children? Anesthesiology 1982;57:340-2.

8. Mauneksela EL, Korpela R. Double-blind evaluation of a lidocaine-prilocaine cream (EMLA) in children: effect on the pain associated with venous cannulation. Br 7 Anaesth 1986;58:1242-5.

9. Arts SE, Abu-Saad HH, Champion GD, Crawford MR, Fisher RJ, Juniper $\mathrm{KH}$, et al. Age-related response to lidocaine-prilocaine (EMLA) emulsion and effect of music distraction on the pain of intravenous cannulation. Pediatrics 1994;93(5):797-801.

10. Manner T, Kanto J, Iisalo E, Lindberg R, Viinamaki O, Scheinin M. Reduction of pain at venous cannulation in children with a eutectic mixture of lidocaine and prilocaine (EMLA cream): comparison with placebo cream and no local premedication. Acta Anaesthesiol Scand 1987;31:735-9.

11. Ehrenstrom Reiz GME, Reiz SLA. EMLA - a eutectic mixture of local anaesthetics for topical anaesthesia. Acta Anaesthes Scad 1982;26:596-8.

12. Waldman SD. Eutectic mixture of local anesthetic (EMLA). Pain Digest 1993; 3:104-9.

13. Bahruth AJ. Peripherally inserted central catheter insertion problems associated with topical anesthesia. 7 Intraven Nurs 1996;19(1):32-4.

14. Taddio A, Gurguis GY, Koren G. Lidocaine-prilocaine cream versus tetracaine gel for procedural pain in children. Ann Pharmacother 2002;36:1-5.

15. Luhmann J, Hurt S, Shootman M, Kennedy R. A comparison of buffered lidocaine versus ELA-Max before peripheral intravenous catheter insertions in children. Pediatrics 2004;113(3):e217-20.

16. Package insert. ELA-Max. Ferndale, MI: Ferndale Laboratories Inc., 2002.

17. Bucalo BD, Mirikitani EJ, Moy RL. Comparison of skin anesthetic effect of liposomal lidocaine, nonliposomal lidocaine, and EMLA using 30-minute application time. Dermatol Surg 1998;24:537-41.

18. Taddio A, Ohlsson A, Einarson T, Stevens B, Koren G. A systematic review of lidocaine-prilocaine cream (EMLA) in the treatment of acute pain in neonates. Pediatrics 1998;101(2)e1.

19. Eichenfield LF, Funk A, Fallon-Friedlander S, Cunningham BB. A clinical study to evaluate the efficacy of ELA-Max (4\% liposomal lidocaine) as compared with eutectic mixture of local anesthetics cream for pain reduction of venipuncture in children. Pediatrics 2002;109:1093-9.

20. Kleiber C, Sorenson M, Whiteside K, Gronstal BA, Tannous R. Topical anesthetics for intravenous insertion in children: a randomized equivalency study. Pediatrics 2002;110(4):758-61.

21. Koh JL, Harrison D, Myers R, Dembinski R, Turner H, McGraw T. A randomized, double-blind comparison study of EMLA and ELA-Max for topical anesthesia in children undergoing intravenous insertion. Pediatr Anaesth 2004;14:977-82

22. Hicks CL, von Baeyer CL, Spafford PA, van Korlaar I, Goodenough B. The faces pain scale-revised: toward a common metric in pediatric pain measurement. Pain 2001;93:173-83.

23. Schulz KF, Grimes DA. Sample size slippages in randomised trials: exclusions and the lost and wayward. Lancet 2002;359:781-5.

24. West N, Oakes L, Hinds PS, Sanders L, Holden R, Williams S, et al. Measuring pain in pediatric oncology ICU patients. 7 Pediatr Oncol Nurs 1994;11:64-8.

25. Wong DL, Baker CM. Pain in children: comparison of assessment scales. $P e-$ diatr Nurs 1988;14:9-17.

26. Fogel-Keck J, Gerkensmeyer JE, Joyce BA, Schade JG. Reliability and validity of the faces and word descriptor scales to measure procedural pain. 7 Pediatr Nurs 1996;11:368-74.

27. Bulloch B, Tenebein M. Validation of 2 pain scales for use in the pediatric emergency department. Pediatrics 2002;110(3):33-8.

28. Chen E, Joseph MH, Zeltzer LK. Behavioral and cognitive interventions in the treatment of pain in children. Pediatr Clin NA 2000;47(3):513-25.

29. Kleiber C, Harper DC. Effects of distraction on children's pain and distress during medical procedures: a meta-analysis. Nurs Res 1999;48(1):44-9.

30. Kennedy RM, Luhmann JD. The "ouchless emergency department." Emerg Med 1999;46(6):1215-47.

Correspondence to: Dr. Anna Taddio, Department of Pharmacy, The Hospital for Sick Children, 555 University Ave., Toronto ON M5G $1 \times 8$

\section{Clinical trial registration}

CMAJ will consider clinical trials for publication only if they have been registered in a publicly accessible clinical trials registry before the enrolment of the first patient. This policy applies to trials that start recruiting on or after July 1, 2005. For trials that began enrolment before this date, registration is required by Sept. 13, 2005. The criteria for acceptable registration are described in CMAJ (2005;172[13]:1700-2). 